International Journal of Pure and Applied Mathematics

Volume 82 No. 4 2013, 513-519

ISSN: 1311-8080 (printed version); ISSN: 1314-3395 (on-line version)

url: http://www.ijpam.eu

doi: http://dx.doi.org/10.12732/ijpam.v82i4.1

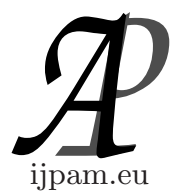

\title{
TUPLE OF OPERATORS WITH THE PROPERTY OF HYPERCYCLICITY CRITERION
}

\author{
B. Yousefi ${ }^{\S}$, Gh.R. Moghimi ${ }^{2}$, M.M. Khorami ${ }^{3}$ \\ ${ }^{1,2,3}$ Department of Mathematics \\ Payame Noor University \\ P.O. Box 19395-4697, Tehran, IRAN
}

\begin{abstract}
In this paper we give the conditions under which a tuple of operators holds in the Hypercyclicity Criterion.
\end{abstract}

AMS Subject Classification: 47B37, 47B33

Key Words: tuple, hypercyclic vector, hypercylicity criterion, hereditarily hypercyclicity

\section{Introduction}

By an $n$-tuple of operators we mean a finite sequence of length $n$ of commuting continuous linear operators on a Banach space $X$.

Definition 1.1. Let $\mathcal{T}=\left(T_{1}, T_{2}, \ldots, T_{n}\right)$ be an $n$-tuple of operators acting on an infinite dimensional Banach space $X$. We will let $\mathcal{F}=\left\{T_{1}{ }^{k_{1}} T_{2}{ }^{k_{2}}, \ldots, T_{n}{ }^{k_{n}}\right.$ : $\left.k_{i} \in \mathbb{Z}_{+}, \quad i=1, \ldots, n\right\}$ be the semigroup generated by $\mathcal{T}$. For $x \in X$, the orbit of $x$ under the tuple $\mathcal{T}$ is the set $\operatorname{Orb}(\mathcal{T}, x)=\{S x: S \in \mathcal{F}\}$. A vector $x$ is called a hypercyclic vector for $\mathcal{T}$ if $\operatorname{Orb}(\mathcal{T}, x)$ is dense in $X$ and in this case the tuple $\mathcal{T}$ is called hypercyclic. Also, by $\mathcal{T}_{d}^{(k)}$ we will refer to the set of all $k$ copies of an element of $\mathcal{F}$, i.e. $\mathcal{T}_{d}^{(k)}=\left\{S_{1} \oplus \ldots \oplus S_{k}: S_{1}=\ldots=S_{k} \in \mathcal{F}\right\}$.

Received: December 12, 2011

(C) 2013 Academic Publications, Ltd. url: www.acadpubl.eu

${ }^{\S}$ Correspondence author 
We say that $\mathcal{T}_{d}^{(k)}$ is hypercyclic provided there exist $x_{1}, \ldots, x_{k} \in X$ such that $\left\{W\left(x_{1} \oplus \ldots \oplus x_{k}\right): W \in \mathcal{T}_{d}^{(k)}\right\}$ is dense in the $k$ copies of $X, X \oplus \ldots \oplus X$.

Note that if $T_{1}, T_{2}, \ldots, T_{n}$ are commutative bounded linear operators on a Banach space $X$, and $\left\{m_{j}(i)\right\}_{j}$, is a sequence of natural numbers for $i=1, \ldots, n$, then we say $\left\{T_{1}^{m_{j}(1)} T_{2}{ }^{m_{j}(2)} \ldots T_{n} m_{j}(n): j \geq 0\right\}$ is hypercyclic if there exists $x \in X$ such that $\left\{T_{1}^{m_{j}(1)} T_{2}{ }^{m_{j}(2)} \ldots T_{n}{ }^{m_{j}(n)} x: j \geq 0\right\}$ is dense in $X$.

Definition 1.2. We say that a tuple $\mathcal{T}=\left(T_{1}, T_{2}, \ldots, T_{n}\right)$ is topologically transitive with respect to a tuple of nonnegative integer sequences

$$
\left(\left\{k_{j(1)}\right\}_{j},\left\{k_{j(2)}\right\}_{j}, \ldots,\left\{k_{j(n)}\right\}_{j}\right),
$$

if for every nonempty open subsets $U, V$ of $X$ there exists $j_{0} \in \mathbb{N}$ such that $T_{1}^{k_{j_{0}(1)}} T_{2}^{k_{j_{0}(2)}} \ldots T_{n}^{k_{j_{0}(n)}}(U) \cap V \neq \varnothing$. Also, we say that an $n$-tuple $\mathcal{T}$ is topologically transitive if it is topologically transitive with respect an $n$-tuple of nonnegative integer sequences.

Definition 1.3. We say that a pair $\mathcal{T}=\left(T_{1}, T_{2}, \ldots T_{n}\right)$ is hereditarily hypercyclic with respect to a tuple of nonnegative increasing sequences

$$
\left(\left\{k_{j(1)}\right\}_{j},\left\{k_{j(2)}\right\}_{j}, \ldots,\left\{k_{j(n)}\right\}_{j}\right)
$$

of integers provided for all tuple of subsequences $\left(\left\{k_{j_{i}(1)}\right\}_{i},\left\{k_{j_{i}(2)}\right\}_{i}, \ldots,\left\{k_{j_{i}(n)}\right\}_{i}\right)$ of $\left(\left\{k_{j(1)}\right\}_{j},\left\{k_{j(2)}\right\}_{j}, \ldots,\left\{k_{j(n)}\right\}_{j}\right)$, the sequence $\left\{T_{1}^{k_{j_{i}(1)}} T_{2}^{k_{j_{i}(2)}} \ldots T_{n}^{k_{j_{i}(n)}}: i \geq 1\right\}$ is hypercyclic. We say that an $n$-tuple $\mathcal{T}$ is hereditarily hypercyclic, if it is hereditarily hypercyclic with respect to an $n$-tuple of nonnegative increasing sequences of integers.

The formulation of the Hypercyclicty Criterion in the next section was given by N. S. Feldman ([5]). Here, we want to extend some properties of hypercyclic operators to a pair of commuting operators, and although the techniques work for any n-tuple of operators but for simplicity we prove our results only for the case $n=2$. For some other topics we refer to [1-18].

\section{Main Results}

In this section we characterize the equivalent conditions for a tuple of operators, satisfying the Hypercyclicity Criterion.

Theorem 2.1. (The Hypercyclicity Criterion) Suppose that $X$ is a separable infinite dimensional Banach space and $\mathcal{T}=\left(T_{1}, T_{2}, \ldots, T_{n}\right)$ be the $n$-tuple 
of operators $T_{1}, T_{2}, \ldots, T_{n}$ acting on $X$. If there exist two dense subsets $Y$ and $Z$ in $X$, and strictly increasing sequences $\left\{m_{j}(i)\right\}_{j}$ for $i=1, \ldots, n$ such that:

1. $T_{1}^{m_{j}(1)} \ldots T_{n}^{m_{j}(n)} y \rightarrow 0$ for all $y \in Y$,

2. there exist a sequence of functions $\left\{S_{j}: Z \rightarrow X\right\}$ such that for every $z \in Z, S_{j} z \rightarrow 0$, and $T_{1}^{m_{j}(1)} \ldots T_{n}^{m_{j}(n)} S_{j} z \rightarrow z$ as $j \rightarrow \infty$.

Then $\mathcal{T}$ is a hypercyclic tuple.

In the proof of the following lemma, we use a method of the proof of Theorem 1.2 in [2] to extend the results for tuples. We will use $\operatorname{HC}(\mathcal{T})$ for the collection of hypercyclic vectors for a tuple of operator $\mathcal{T}$.

Lemma 2.2. Let $X$ be a separable infinite dimensional Banach space and $\mathcal{T}=\left(T_{1}, T_{2}, \ldots, T_{n}\right)$ be the tuple of operators $T_{1}, T_{2}, \ldots, T_{n}$. Then the followings are equivalent:

(i) $\mathcal{T}$ is hypercyclic.

(ii) for all nonempty open subsets $U, V$ in $X$, there exists a tuple of sequences $\left(\left\{k_{j}(1)\right\}_{j},\left\{k_{j}(2)\right\}_{j}, \ldots,\left\{k_{j}(n)\right\}_{j}\right)$ of integers such that for all $j \geq 0$,

$$
T_{1}^{k_{j}(1)} T_{2}{ }^{k_{j}(2)} \ldots T_{n}{ }^{k_{j}(n)}(U) \cap V \neq \varnothing .
$$

(iii) $\mathcal{T}$ is topologically transitive.

Proof. First note that if $x$ is a hypercyclic vector for $\mathcal{T}$, then for all natural numbers $m_{1}, \ldots, m_{n}$, the orbit $\operatorname{Orb}\left(\mathcal{T}, T_{1}^{m_{1}} T_{2}{ }^{m_{2}} \ldots T_{n}{ }^{m_{n}} x\right)$ is equal to

$$
\operatorname{Orb}(\mathcal{T}, x) \backslash\left\{T_{1}{ }^{j_{1}} T_{2}{ }^{j_{2}} \ldots T_{n}{ }^{j_{n}} x: j_{i}=0, \ldots, m_{i}-1 ; i=1, \ldots, n\right\} .
$$

Since $X$ has no isolated point, $\operatorname{Orb}(\mathcal{T}, x)$ remains dense after the removal of a finite number of points. Hence $T_{1}^{m_{1}} T_{2}{ }^{m_{2}} \ldots T_{n}{ }^{m_{n}} x \in H C(\mathcal{T})$ for all $m_{1}, \ldots, m_{n} \in$ $\mathbb{N}$ and so $\operatorname{Orb}(\mathcal{T}, x) \subset H C(\mathcal{T})$. So $H C(\mathcal{T})$ is dense or empty.

(i) $\rightarrow$ (ii): Let $\mathcal{T}$ be hypercyclic and $(U, V)$ be a pair of nonempty open subsets of $X$. Since $H C(\mathcal{T})$ is dense, thus the sets $U \cap H C(\mathcal{T})$ and $V \cap$ $H C(\mathcal{T})$ are nonempty. Choose $x \in U \cap H C(\mathcal{T})$ and $y \in V \cap H C(\mathcal{T})$, and pick $k_{0}$ such that $B\left(y, \frac{1}{k_{0}}\right) \subset V$. Since $B\left(y, \frac{1}{k}\right) \cap \operatorname{Orb}(\mathcal{T}, x)$ is nonempty, thus there there exists a tuple $\left(m_{k}^{\prime}(1), m_{k}^{\prime}(2), \ldots, m_{k}^{\prime}(n)\right)$ of integers such that $T_{1}^{m_{k}^{\prime}(1)} T_{2}{ }^{m_{k}^{\prime}(2)} \ldots T_{n}{ }^{m_{k}^{\prime}(n)}(x) \in B\left(y, \frac{1}{k}\right) \cap H C(\mathcal{T})$, and so

$$
T_{1}^{m_{k}^{\prime}(1)} T_{2}^{m_{k}^{\prime}(2)} \ldots T_{n}{ }^{m_{k}^{\prime}(n)}(U) \cap V \neq \varnothing
$$


for all $k \geq k_{0}$. Define $m_{k}(i)=m_{k_{0}+k}^{\prime}(i)$ for all $i=1, \ldots, n$ and for all $k \geq 0$. Then $T_{1}^{m_{k}(1)} T_{2}{ }^{m_{k}(2)} \ldots T_{n}{ }^{m_{k}(n)}(U) \cap V \neq \varnothing$ for all $k \geq 0$.

(ii) $\rightarrow$ (iii): it is clear.

(iii) $\rightarrow$ (i): Fix an enumeration $\left\{B_{n}: n \in \mathbb{N}\right\}$ of the open balls in $X$ with rational radii, and centers in a countable dense subset of $X$. By the continuity of the operators $T_{1}, T_{2}, \ldots, T_{n}$, the sets

$$
G_{k}=\bigcup\left\{T_{1}^{-m(1)} T_{2}^{-m(2)} \ldots T_{n}^{-m(n)}\left(B_{k}\right): m(i) \geq 0, \quad i=1, \ldots, n\right\}
$$

are open. Clearly $\mathrm{HC}(\mathcal{T})$ is equal to $\bigcap\left\{G_{k}: k \in \mathbb{N}\right\}$. Now let $\mathcal{T}$ be topologically transitive and let $W$ be an arbitrary nonempty open set in $X$. Then for all $i=1, \ldots, n$ and $k \in \mathbb{N}$, there exists $m_{k}(i)$ in $\mathbb{N}$ such that

$$
T_{1}^{m_{k}(1)} T_{2}^{m_{k}(2)} \ldots T_{n}^{m_{k}(n)}(W) \cap B_{k} \neq \varnothing
$$

which implies that $W \cap G_{k} \neq \varnothing$ for all $k$. Thus each $G_{k}$ is dense in $X$ and so by the Bair Category Theorem $H C(\mathcal{T})$ is also dense in $X$. In particular, $H C(\mathcal{T})$ is nonempty and so (i) holds.

Corollary 2.3. $\mathcal{T}_{d}^{(2)}$ is hypercyclic if and only if for given four nonempty open subsets $U_{1}, U_{2}, V_{1}, V_{2}$ of $X$, there exists a tuple of integers $(m(1), \ldots, m(n))$ such that

$$
T_{1}^{m(1)} T_{2}^{m(2)} \ldots T_{n}^{m(n)}\left(U_{1}\right) \cap V_{1} \neq \varnothing ; \quad T_{1}^{m(1)} T_{2}^{m(2)} \ldots T_{n}^{m(n)}\left(U_{2}\right) \cap V_{2} \neq \varnothing .
$$

Theorem 2.4. Let $\mathcal{T}=\left(T_{1}, T_{2}, \ldots, T_{n}\right)$ be a tuple of operators acting on a separable infinite dimensional Banach space $X$. Then the followings are equivalent:

(i) $\mathcal{T}$ satisfies the Hypercyclicity Criterion.

(ii) $\mathcal{T}$ is hereditarily hypercyclic.

(iii) $\mathcal{T}_{d}^{(2)}$ is hypercyclic.

Proof. (i) $\rightarrow$ (ii): Let $X_{0}, Y_{0},\left(\left\{m_{j}(1)\right\}_{j},\left\{m_{j}(2)\right\}_{j}, \ldots,\left\{m_{j}(n)\right\}_{j}\right)$, and $S_{j}: Y_{0} \longrightarrow X$ be as given in the Hypercyclicity Criterion. Notice that Hypercyclicity Criterion will also be satisfied by any tuple of subsequences

$$
\left.\left(\left\{m_{j_{k}}(1)\right\}_{k},\left\{m_{j_{k}}(2)\right\}\right)_{k}, \ldots,\left\{m_{j_{k}}(n)\right\}_{k}\right)
$$


of $\left(\left\{m_{j}(1)\right\}_{j},\left\{m_{j}(2)\right\}_{j}, \ldots,\left\{m_{j}(n)\right\}_{j}\right)$. Hence, it suffices to check that

$$
\left\{T_{1}{ }^{m_{j}(1)} T_{2}{ }^{m_{j}(2)} \ldots T_{n}{ }^{m_{j}(n)}: j \geq 1\right\}
$$

is hypercyclic that is equivalent to that $\mathcal{T}$ is hereditarily hypercyclic with respect to the tuple $\left(\left\{m_{j}(1)\right\}_{j},\left\{m_{j}(2)\right\}_{j}, \ldots,\left\{m_{j}(n)\right\}_{j}\right)$. Now let $U$ and $V$ be nonempty open subsets of $X$ and pick $x \in X_{0}, y \in Y_{0}$ and $\epsilon>0$ so that $B(x, \epsilon) \subset U$ and $B(y, 2 \epsilon) \subset V$. By the conditions of Hypercyclicity Criterion, there exist $r$ arbitrary large satisfying:

$$
T_{1}^{m_{r}(1)} T_{2}^{m_{r}(2)} \ldots T_{n}^{m_{r}(n)} x \in B(0, \epsilon), \quad S_{r} y \in B(0, \epsilon),
$$

and

$$
T_{1}^{m_{r}(1)} T_{2}^{m_{r}(2)} \ldots T_{n}^{m_{r}(n)} S_{r} y-y \in B(0, \epsilon) .
$$

So we get $x+S_{r} y \in B(x, \epsilon) \subset U$ and

$$
\left\|T_{1}^{m_{r}(1)} T_{2}^{m_{r}(2)} \ldots T_{n}^{m_{r}(n)} S_{r} y-y\right\|+\left\|T_{1}^{m_{r}(1)} T_{2}^{m_{r}(2)} \ldots T_{n}^{m_{r}(n)} x\right\|<2 \epsilon .
$$

Thus $T_{1}^{m_{r}(1)} T_{2}^{m_{r}(2)} \ldots T_{n}^{m_{r}(n)} S_{r} y-y \in B(y, 2 \epsilon) \subset V$. Hence

$$
T_{1}^{m_{r}(1)} T_{2}^{m_{r}(2)} \ldots T_{n}^{m_{r}(n)}(U) \cap V
$$

is nonempty and so by the Lemma 2.2, $\left\{T_{1}{ }^{m_{j}(1)} T_{2}{ }^{m_{j}(2)} \ldots T_{n}{ }^{m_{j}(n)}: j \geq 1\right\}$ is hypercyclic which implies that $\mathcal{T}$ is hereditarily hypercyclic with respect to $\left(\left\{m_{j}(1)\right\}_{j},\left\{m_{j}(2)\right\}_{j}, \ldots,\left\{m_{j}(n)\right\}_{j}\right)$.

(ii) $\rightarrow$ (iii): Suppose $\mathcal{T}$ is hereditarily hypercyclic with respect to the tuple of sequences $\left(\left\{m_{j}(1)\right\}_{j},\left\{m_{j}(2)\right\}_{j}, \ldots,\left\{m_{j}(n)\right\}_{j}\right)$ and for $i=1,2$, let $\left(U_{i}, V_{i}\right)$ be a pair of nonempty open subsets of $X$. We want to show that there are arbitrary large positive integers $m_{1}, \ldots, m_{n}$ satisfying $T_{1}^{m_{1}} T_{2}^{m_{2}} \ldots T_{n}^{m_{n}}\left(U_{i}\right) \cap V_{i} \neq \varnothing$ for $i=1,2$. Since $\left\{T_{1}{ }^{m_{j}(1)} T_{2}{ }^{m_{j}(2)} \ldots T_{n}{ }^{m_{j}(n)}: j \geq 1\right\}$ is hypercyclic, by Lemma 2.2., there exists a tuple of subsequences $\left.\left(\left\{m_{j_{k}}(1)\right\}_{k},\left\{m_{j_{k}}(2)\right\}\right)_{k}, \ldots,\left\{m_{j_{k}}(n)\right\}_{k}\right)$ of $\left(\left\{m_{j}(1)\right\}_{j},\left\{m_{j}(2)\right\}_{j}, \ldots,\left\{m_{j}(n)\right\}_{j}\right)$ with $T_{1}^{m_{j_{k}}(1)} T_{2}^{m_{j_{k}}(2)} \ldots T_{n}^{m_{j_{k}}(n)}\left(U_{1}\right) \cap V_{1} \neq \varnothing$ for $j \geq 1$. But $\mathcal{T}$ is hereditarily hypercyclic with respect to $\left(\left\{m_{k}\right\},\left\{n_{k}\right\}\right)$, thus $\left\{T_{1}^{m_{j_{k}}(1)} T_{2}^{m_{j_{k}}(2)} \ldots T_{n}^{m_{j_{k}}(n)}: k \geq 1\right\}$ is also hypercyclic. Hence, by Lemma 2.2, for $i=1, \ldots, n$, there exists $m_{i} \in\left\{m_{j_{k}}(i)\right\}$ arbitrary large so that

$$
T_{1}^{m_{1}} T_{2}^{m_{2}} \ldots T_{n}^{m_{n}}\left(U_{2}\right) \cap V_{2} \neq \varnothing .
$$

Hence we get $T_{1}^{m_{1}} T_{2}^{m_{2}} \ldots T_{n}^{m_{n}}\left(U_{i}\right) \cap V_{i} \neq \varnothing$ for $i=1,2$, and so $\mathcal{T}_{d}^{(2)}$ is hypercyclic and (iii) holds. 
(iii) $\rightarrow$ (i): Let $x \oplus y$ be a hypercyclic vector for $\mathcal{T}_{d}^{(2)}$. In particular, $x$ and $y$ are hypercyclic for $\mathcal{T}$. Thus for all tuple of nonnegative integers $\left(m_{1}, m_{2}, \ldots, m_{n}\right)$, the vector $T_{1}^{m_{1}} T_{2}^{m_{2}} \ldots T_{n}^{m_{n}} y$ is hypercyclic for $\mathcal{T}$ and so $\left(x, T_{1}^{m_{1}}\right.$ $\left.T_{2}^{m_{2}} \ldots T_{n}^{m_{n}} y\right)$ is also a hypercyclic vector for $\mathcal{T}_{d}^{(2)}$. This implies that for all nonempty open subset $U$ of $X$, there is $u \in U$ such that $(x, u)$ is a hypercyclic vector for $\mathcal{T}_{d}^{(2)}$. Fix now $\left\{U_{k}\right\}_{k \geq 1}$ a decreasing 0-basis in $X$. Proceeding by induction we find $u_{j} \in U_{j}$ for all $j \in \mathbb{N}$, and increasing sequences $\left\{m_{j}(i)\right\}_{j}$ $(i=1, \ldots, n)$ of natural numbers satisfying $T_{1}^{m_{j}(1)} T_{2}{ }^{m_{j}(2)} \ldots T_{n}{ }^{m_{j}(n)} x \in U_{j}$ and

$$
T_{1}^{m_{j}(1)} T_{2}^{m_{j}(2)} \ldots T_{n}^{m_{j}(n)} u_{j} \in x+U_{j}
$$

for all $j \in \mathbb{N}$. Let $X_{0}=\operatorname{Orb}(\mathcal{T}, x)$ which is dense in $X$. Then we have that $T_{1}^{m_{j}(1)} T_{2}{ }^{m_{j}(2)} \ldots T_{n}{ }^{m_{j}(n)} x \rightarrow 0$ and so $T_{1}^{m_{j}(1)} T_{2}{ }^{m_{j}(2)} \ldots T_{n}{ }^{m_{j}(n)} v \rightarrow 0$ for all $v \in$ $X$. Define $S_{j}\left(T_{1}^{m_{1}} T_{2}^{m_{2}} \ldots T_{n}^{m_{n}}\right) x=T_{1}^{m_{1}} T_{2}^{m_{2}} \ldots T_{n}^{m_{n}} u_{j}$ for all $m_{i}, i=1, \ldots, n$, and all $j$ in $\mathbb{N}$. Then $S_{j} v \rightarrow 0$ for all $v \in X_{0}$. Finally, given $m_{0}(1), m_{0}(2), \ldots, m_{0}(n) \in$ $\mathbb{N}$, we get that

$$
T_{1}^{m_{j}(1)} T_{2}^{m_{j}(2)} \ldots T_{n}^{m_{j}(n)} S_{j}\left(T_{1}^{m_{0}(1)} T_{2}^{m_{0}(2)} \ldots T_{n}^{m_{0}}(n) x\right)
$$

is equal to

$$
T_{1}^{m_{0}(1)} T_{2}^{m_{0}(2)} \ldots T_{n}^{m_{0}}(n)\left(T_{1}^{m_{j}(1)} T_{2}^{m_{j}(2)} \ldots T_{n}^{m_{j}(n)} u_{j}\right)
$$

which tends to $T_{1}^{m_{0}(1)} T_{2}^{m_{0}(2)} \ldots T_{n}^{m_{0}}(n) x$ as $j \rightarrow \infty$. This completes the proof.

\section{References}

[1] F. Bayart, E. Matheron, Dynamics of Linear Operators, Cambridge University Press (2009).

[2] Bernal-Gonzales, K.G. Gross-Erdmann, The hypercyclicity criterionfor sequences of operators, Studia Math., 157 (2003), 45-54.

[3] J. Bes, A. Peris, Hereditarily hypercyclic operators, J. Func. Anal., 167 (1) (1999), 94-112.

[4] N. S. Feldman, Hypercyclic pairs of coanalytic Toeplitz operators, Integral Equations Operator Theory, 58, No. 20 (2007), 153-173.

[5] N. S. Feldman, Hypercyclic tuples of operators and somewhere dense orbits, J. Math. Appl., 346 (2008), 82-98. 
[6] G. Godefroy and J. H. Shapiro, Operators with dense invariant cyclic manifolds, J. Func. Anal., 98 (1991), 229-269.

[7] S. Grivaux, Hypercyclic operators, mixing operators and the bounded steps problem, J. Operator Theory, 54 (2005), 147-168.

[8] S. Yarmahmoodi, K. Hedayatian and B. Yousefi, Supercyclicity and hypercyclicity of an isometry plus a nilpotent, Abstract and Applied Analysis, 2011 (2011), doi: 10.1155/2011/686832.

[9] B. Yousefi, and H. Rezaei, Hypercyclicity on the algebra of Hilbert-Schmidt operators, Results in Mathematics, 46 (2004), 174-180.

[10] B. Yousefi and H. Rezaei, Some necessary and sufficient conditions for Hypercyclicity Criterion, Proc. Indian Acad. Sci. (Math. Sci.), 115, No. 2 (2005), 209-216.

[11] B. Yousefi and A. Farrokhinia, On the hereditarily hypercyclic vectors, Journal of the Korean Mathematical Society, 43, No. 6 (2006), 1219-1229.

[12] B. Yousefi, H. Rezaei and J. Doroodgar, Supercyclicity in the operator algebra using Hilbert-Schmidt operators, Rendiconti Del Circolo Matematico di Palermo, Serie II, Tomo LVI (2007), 33-42.

[13] B. Yousefi and H. Rezaei, Hypercyclic property of weighted composition operators, Proc. Amer. Math. Soc., 135, No. 10 (2007), 3263-3271.

[14] B. Yousefi and S. Haghkhah, Hypercyclicity of special operators on Hilbert function spaces, Czech. Math. Journal, 57, No. 132 (2007), 1035-1041.

[15] B. Yousefi and H. Rezaei, On the supercyclicity and hypercyclicity of the operator algebra, Acta Mathematica Sinica, 24, No. 7 (2008), 1221-1232.

[16] B. Yousefi and R. Soltani, Hypercyclicity of the adjoint of weighted composition operators, Proc. Indian Acad. Sci. (Math. Sci.), 119, No. 3 (2009), 513-519.

[17] B. Yousefi and J. Izadi, Weighted composition operators and supercyclicity criterion, International Journal of Mathematics and Mathematical Sciences, Volume 2011 (2011), doi: 10.1155/2011/514370.

[18] B. Yousefi, Hereditarily transitive tuples, Rend. Circ. Mat. Palermo, 2011 (2011), doi: 10.1007/S12215-011-0066-y. 
